\title{
Anti-Obesity Effect of Lactobacillus plantarum LB818 Is Associated with Regulation of Gut Microbiota in High-Fat Diet-Fed Obese Mice
}

\author{
Ahtesham Hussain, ${ }^{1}$ Mi Hyun Kwon, ${ }^{1}$ Hyun Kyu Kim, ${ }^{2}$ Hak Sung Lee, ${ }^{2}$ Jin Sook Cho, ${ }^{1}$ and Young Ik Lee ${ }^{1}$ \\ ${ }^{1}$ Lee's Biotech Co., Ltd., Daejeon, South Korea. \\ ${ }^{2}$ Kolmar, BNH Co., Ltd., Sejong-si, Korea.
}

\begin{abstract}
Worldwide, obesity has become a major risk factor associated with health risks such as diabetes, hypertension, hypercholesterolemia, cardiovascular disease, stroke, and certain forms of cancer. In this study, we estimated the anti-obesity effect of the bacterial strain Lactobacillus plantarum LB818 (designated as LB818) using male C57BL/6J mice, which were treated with high-fat diet (HFD) to induce obesity. Next, LB818 $\left(10^{9}\right.$ colony-forming units $\left.[\mathrm{CFU}] / \mathrm{mL}\right)$ was orally administered for 8 weeks. The results showed that feeding HFD+LB818 $\left(10^{9} \mathrm{CFU} / \mathrm{mL}\right)$ ameliorated body weight gain and decreased total body fat by regulating fasting glucose levels in HFD-fed mice. LB818 treatment significantly lowered aspartate aminotransferase, alanine aminotransferase (ALT), total cholesterol (TC), triglyceride (TG), and elevated high-density lipoprotein levels in serum and decreased deposition of fat droplets in liver. LB818 treatment increased the respective abundances of essential bacteria, including Bacteroidetes, Akkermansia, Bifidobacterium, Lactobacillus, and increased the Bacteroidetes:Firmicutes ratio; however, it significantly decreased the levels of Firmicutes. Taken together, this study demonstrates that LB818 is effective in attenuating obesity and hepatic steatosis and regulated gut microbiota in HFD-fed obese mice.
\end{abstract}

KEYWORDS: • anti-obesity $\bullet$ gut microbiota $\bullet$ high-fat diet $\bullet$ Lactobacillus plantarum LB818

\section{INTRODUCTION}

O BESITY AND DIABETES are major health problems. Obesity occurs owing to immoderate consumption of foods rich in fats and carbohydrates, which causes disparity between energy uptake and energy expenditure. ${ }^{1}$ It is related to various complications of life-threatening diseases, including cardiovascular diseases, hypertension, diabetes, and certain form of cancers, and is additionally related to hyperglycemia, insulin resistance, increase cholesterol level, and hepatic steatosis. ${ }^{1,2}$

The development of obesity and associated metabolic disorders is related to alterations in gut microbiota. ${ }^{3-5}$ Host metabolism is affected by alterations in the gut microbiota, which in turn has an impact on energy homeostasis and ectopic fat deposition. ${ }^{6}$ Two microbial communities represent gut microbiota, which predominantly include Firmicutes (e.g., Ruminococcus, Clostridium, Eubacterium, and Lactobacillus), Bacteroidetes (e.g., Prevotella, Bacteroides), Actinobacteria (Bifidobacterium), and Proteobacteria. ${ }^{7}$ Accumulating evidence indicates that gut microbiota play a major role in human obesity by regulating lipid and glucose

Manuscript received 07 November 2019. Revision accepted 08 April 2020.

Address correspondence to: Young Ik Lee, PhD, Lee's Biotech Co., Ltd., \#416, C Dong, 17 Techno 4-ro, Yuseon-gu, Daejeon 34013, South Korea, E-mail: yilee@kribb.re.kr metabolism and influencing energy extraction, inflammation, and neuroendocrine secretions. ${ }^{8-10}$ Most beneficial microorganisms, identified as probiotics to date, are beneficial to human health. Such beneficial microorganisms belong to the genera of Lactobacillus and Bifidobacterium and act by improving the immune system, preventing cancer, augmenting intestinal functions, and exhibiting hypocholesterolemic effect. ${ }^{11-15}$ Numerous studies have demonstrated the nutritional benefits and potential health-promoting effects of lactobacilli, including those on the development of obesity and related metabolic disorders, including diabetes, hypertension, and hypercholesterolemia. ${ }^{16-20}$

Overall, the aim of this study was to evaluate the preventive effects of the probiotic strain Lactobacillus plantarum LB818 (designated LB818) on fat accumulation in adipose tissue and hepatic steatosis in HFD-fed obese mice, and to examine its efficacy in restoring gut microbiota in diet-induced obese mice.

\section{MATERIALS AND METHODS}

\section{Statement of ethics}

The animals were accommodated and cared for in accordance to the National Institute of Health instruction for taking care and use of laboratory animals. The animal study protocols were approved by the Institute Animal Ethics 
Committee, Korea Research Institute of Bioscience and Biotechnology (KRIBB-AEC-18176) Daejeon, Korea.

\section{Isolation of $L B 818$}

LB818 was isolated from locally fermented vegetables (Kimchi) and was grown in de Man, Rogosa, and Sharpe (MRS) broth at $37^{\circ} \mathrm{C}$ for $20-24 \mathrm{~h}$. Kimchi was blended with $0.85 \% \mathrm{NaCl}$ and serially diluted and then spread on MRS agar plates and incubated for $24 \mathrm{~h}$ at $37^{\circ} \mathrm{C}$. Representative colonies were picked from plates with the highest dilution that displayed colonies. After purification, the strain was stored at $-70^{\circ} \mathrm{C}$. Strains were identified by $16 \mathrm{~S}$ rRNA sequencing analysis that was performed using a kit procured from Macrogen (Korea) and analyzed using the BLAST database.

\section{Acid tolerance test for LB818}

Bacteria were grown in MRS broth medium for $24 \mathrm{~h}$ at $37^{\circ} \mathrm{C}$. Then cultures were diluted with phosphate-buffered saline and maintained with $\mathrm{pH}$ values of 3.0 or 2.0 , adjusted using $0.5 \mathrm{~N} \mathrm{HCl}$ to $\sim 10^{8}$ colony-forming units $(\mathrm{CFU}) / \mathrm{mL}$. Next, the broth was incubated at $37^{\circ} \mathrm{C}$ for $120 \mathrm{~min}$, and the viable bacterial count was determined at various time points $(0,20,40,60,80,100$, and $120 \mathrm{~min})$. Cell survival rate was determined according to the total number of colonies grown on MRS agar plates as compared with starting bacterial colonies.

\section{Bile salts tolerance test for LB818}

The potential of LB818 to grow in the existence of various concentrations of bile salts (w/v) $(0 \%, 0.1 \%, 0.3 \%$, $0.5 \%$, and $0.7 \%$, respectively) was tested by inoculating the bacteria ( $1 \%$ of an overnight culture) in MRS broth medium at $37^{\circ} \mathrm{C}$ for $24 \mathrm{~h}$ under aerobic environment. The serially diluted culture was spread on MRS agar plates, and the plates were incubated at $37^{\circ} \mathrm{C}$ for $24 \mathrm{~h}$ to enumerate the surviving bacteria.

\section{Heat resistance test for $L B 818$}

Thermal viability of LB818 during heat stress was tested. Toward this objective, bacteria of the LB818 strain were cultured in MRS broth for $24 \mathrm{~h}$ at $37^{\circ} \mathrm{C}$. Later, the bacterial cultures were heat treated at various temperatures $\left(30^{\circ} \mathrm{C}\right.$, $40^{\circ} \mathrm{C}, 50^{\circ} \mathrm{C}, 60^{\circ} \mathrm{C}$, and $70^{\circ} \mathrm{C}$, respectively) for 15 or $30 \mathrm{~min}$ and were subsequently cooled in an ice box. The samples were serially diluted and spread on MRS agar plates. Bacterial survival rate was determined according to the total number of colonies developed on MRS agar as compared with starting bacterial colonies.

\section{Animal experiments}

A 6-week-old male C57BL/6 mice were purchased from Saeronbio (Gyeonggi-doi, Korea) and acclimatized for 1 week earlier to conducting the animal study. All mice were housed in an environmental room with a temperature of
$21 \pm 2^{\circ} \mathrm{C}$ and relative humidity of $40-60 \%$, and maintained under a $12 \mathrm{~h}$ light and $12 \mathrm{~h}$ dark cycles. The mice were continued on a high-fat diet (HFD), except those in the normal group (provided with a normal chow diet), for 8 weeks. Next, the mice were separated into 4 groups, a normal group (normal diet [ND]; $n=6$ ), HFD only group $(n=6)$, HFD+simvastatin (SMV; $10 \mathrm{mg} / \mathrm{kg}$ body weight administered orally, $n=6$; positive control), HFD+LB818 $\left(10^{9} \mathrm{CFU} / \mathrm{mL}\right)$. The normal chow diet consisted of $12.7 \%$ calories obtained from fat, whereas the HFD comprised $60 \%$ calories obtained from fat. Body weights of the animals were measured weekly. Mice were maintained for 8 weeks only on HFD and for a further 8 weeks on treatments as mentioned previously. Ultimately, after $14-16 \mathrm{~h}$ of fasting, blood samples were collected from the mice retro-orbital vein before the animals were killed to estimate serum biochemical parameters and fasting blood sugar. For histological analysis of liver and fat tissues, the removed tissues were washed quickly, established in $10 \%$ neutral-buffered formaldehyde, and kept until used.

\section{Serum biochemistry measurements}

The collected blood samples were kept for $1 \mathrm{~h}$ at room temperature to allow clotting; the serum samples was separated by centrifugation at $3000 \mathrm{rpm}$ for $15 \mathrm{~min}$ and stored at $-70^{\circ} \mathrm{C}$ until used (Hanil Science Ind. Centrifuge; model MF80). High-density lipoproteins (HDLs), triglycerides (TGs), total cholesterol (TC), low-density lipoproteins (LDLs), aspartate aminotransferase (AST), and alanine aminotransferase (ALT) levels were measured as per the manufacturer's directions and analyzed using the Beckman Coulter AU480 Chemistry Analyzer.

\section{Fasting blood sugar}

After the desired treatments, following $16 \mathrm{~h}$ of fasting, blood was collected from the tail veins and fasting blood sugar were measured using a blood glucose meter (AccuChek Active, Roche, Germany).

\section{Histological analysis}

Liver tissues were fixed in FSC 22 frozen section compound (Leica Biosystems, Richmond, VA, USA) and were sectioned (5 $\mu \mathrm{m}$ thickness) using a cryostat (Tissue-Tek ${ }^{\circledR}$ Cryo3 ${ }^{\circledR}$ Flex Microtome/Cryostat; Sakura). The sections were stained with Oil Red-O stain to analyze fat deposition in liver tissues. Liver tissues set in $10 \%$ neutral-buffered formaldehyde were fixed in paraffin and sectioned at $5 \mu \mathrm{m}$ thickness (Accu-Cut ${ }^{\circledR}$ SRM $^{\mathrm{TM}}$ 200; Sakura). The liver tissue sections were later deparaffinized in xylene, rehydrated in ascending order of ethanol series, stained with hematoxylin and eosin (H\&E), and mounted using a xylene-based medium. Next, the tissue section slides were examined through Olympus BX 61 microscope (Olympus, Tokyo, Japan) at $200 \times$ magnification, and images were obtained using an Olympus DP70 digital camera (Olympus). 
Table 1. List of Bacteria Primers Used in this Study

\begin{tabular}{ll}
\hline Bacteria & \multicolumn{1}{c}{ Primer sequences } \\
\hline Bacteroidetes & 5'-GGA RCA TGT GGT TTA ATT CGA TGA T-3' \\
& 5'-AGC TGA CGA CAA CCA TGC AG-3' \\
Firmicutes & 5'-GGA GYA TGT GGT TTA ATT CGA AGC A-3' \\
& 5'-AGC TGA CGA CAA CCA TGC AC-3' \\
Akkermansia & 5'-CAG CAC GTG AAG GTG GGG AC-3' \\
& 5'-CCT TGC GGT TGG CTT CAG AT-3' \\
Lactobacillus & 5'-GAG GCA GCA GTA GGG AAT CTT C-3' \\
& 5'-GGC CAG TTA CTA CCT CTA TCC TTC TTC-3' \\
Bifidobacterium & 5'-CGC GTC TGG TGT CAA AG-3' \\
& 5'-CCC CAC ATC CAG CAT CCA-3' \\
\hline
\end{tabular}

Determination of bacterial abundance in stool samples using quantitative reverse transcription polymerase chain reaction

After collection, fresh feces samples were kept at $-70^{\circ} \mathrm{C}$ until analysis. Stool DNA was isolated from the samples using an AccuPrep stool DNA extraction kit (BIONEER, Daejeon, Korea) in accordance with the manufacturer's direction. The purity of DNA was determined using NanoDrop $^{\mathrm{TM}}$ (Thermo Fisher Scientific, Wilmington, DE, USA), and the quality of DNA was further evaluated using agarose gel electrophoresis. Quantitative reverse transcription polymerase chain reaction (qRT-PCR) analysis of the stool DNA samples was carried out in an entire reaction mixture volume of $20 \mu \mathrm{L}$ that contained $10 \mu \mathrm{L}$ SYBR Green (master mix), and $1 \mu \mathrm{g}$ of DNA. Table 1 shows the bacterial $16 \mathrm{~S}$ rRNA primers used in this study. The concentrations of DNA, annealing temperature, and primers were optimized as previously mentioned. ${ }^{21}$ Investigation of the data and calculation of $c_{t}$ value were carried out by Light Cycler software (Roche Applied Science). The relative abundance of bacterial DNA was represented using the $2^{-\Delta \mathrm{ct}}$ procedure, and results expressed as normalized fold values of the treatment groups relative to those of the normal group were determined.

\section{Statistical analysis}

All values were presented as mean \pm standard deviation. Statistical significance difference compared with HFD-fed groups was calculated using Student's $t$-test. Values of $P<.05$ were considered statistically significant.

\section{RESULTS}

\section{Acid tolerance effect of LB818}

Bacteria of the LB818 strain and other commercially available lactobacilli were incubated in an acidic environment ( $\mathrm{pH}$ values of 3.0 or 2.0 , adjusted using $0.5 \mathrm{~N} \mathrm{HCl}$ ) for $120 \mathrm{~min}$ at $37^{\circ} \mathrm{C}$. All the bacterial strains showed resistance to acid, including Lactobacillus rhamnosus Mei, LB818, Lactobacillus johnsonii, Lactobacillus reuteri, L. plantarum, and Lactobacillus salivarius at $\mathrm{pH} 3.0$, whereas at $\mathrm{pH} 2.0$ all strains demonstrated strong resistance to acid, except Lactobacillus rhamnosus Mei (Fig. 1A, B). Viable bacteria were
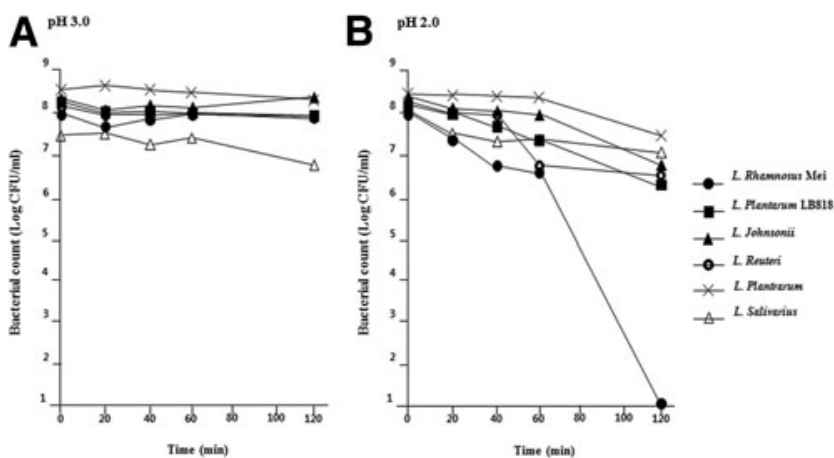

FIG. 1. Effect of acid tolerance on Lactobacillus rhamnosus Mei, Lactobacillus plantarum LB818, Lactobacillus johnsonii, Lactobacillus reuteri, L. plantarum, Lactobacillus salivarius at $120 \mathrm{~min}$, the survival rate of LP LB818 was similar to that of other bacteria at $\mathrm{pH}$ 3.0 (A), whereas there was a decrease in the survival rate of LP LB818 at pH 2 (B) compared with that in other bacteria. Data are shown as numbers of surviving bacteria against time. CFU, colonyforming units; LP, Lactobacillus plantarum.

counted at various time points and cell survival rate was determined according to the number of colonies developed on MRS agar plates compared with initial bacterial concentration.

\section{Effects of bile salts on LB818}

Bacteria were grown in the presence of various concentrations of bile salts $(0 \%, 0.1 \%, 0.3 \%, 0.5 \%$, and $0.7 \%)$ along with other commercially available lactobacilli, such as Lactobacillus rhamnosus Mei and L. johnsonii. At $0 \%$, $0.1 \%$, and $0.3 \%$ of bile salts, all the bacteria survived; however, at $0.1 \%$ and $0.3 \%$ of bile salts, the viable bacterial counts were decreased. At $0.5 \%$ of bile salt, the bacterial cell counts of the LB818 and L. johnsonii strains decreased consistently, whereas those of Lactobacillus rhamnosus Mei had completely died. However, at $0.7 \%$ of bile salts, the LB818 bacteria survived, although with a slight decrease in bacterial cell count (Fig. 2).

\section{Effects of various temperatures on LB818}

Bacteria of the LB818, Lactobacillus rhamnosus Mei, and L. johnsonii strains were heat treated at $30-70^{\circ} \mathrm{C}$ for 15 and 30 min in MRS broth. The results showed that survival rate of all the three bacteria were similar after heat treatment at $30^{\circ} \mathrm{C}, 40^{\circ} \mathrm{C}$, and $50^{\circ} \mathrm{C}$; however, the viable cell count decreased after exposure to higher temperatures $\left(60^{\circ} \mathrm{C}\right.$ and $\left.70^{\circ} \mathrm{C}\right)$. Moreover, after heat treatment at temperature $60^{\circ} \mathrm{C}$ and $70^{\circ} \mathrm{C}$ for 15 and $30 \mathrm{~min}$, respectively, the bacterial survival rate was significantly $(P<.05)$ decreased as compared with the temperature at $30^{\circ} \mathrm{C}$ but similar to all the three bacterial strains (Fig. 3A-C).

Treatment with LB818 decreases total body weight, liver weight, and fat tissue weight in HFD-induced obese mice

Total body weight, checked weekly, was elevated at a higher rate in HFD-fed animals compared with that in 


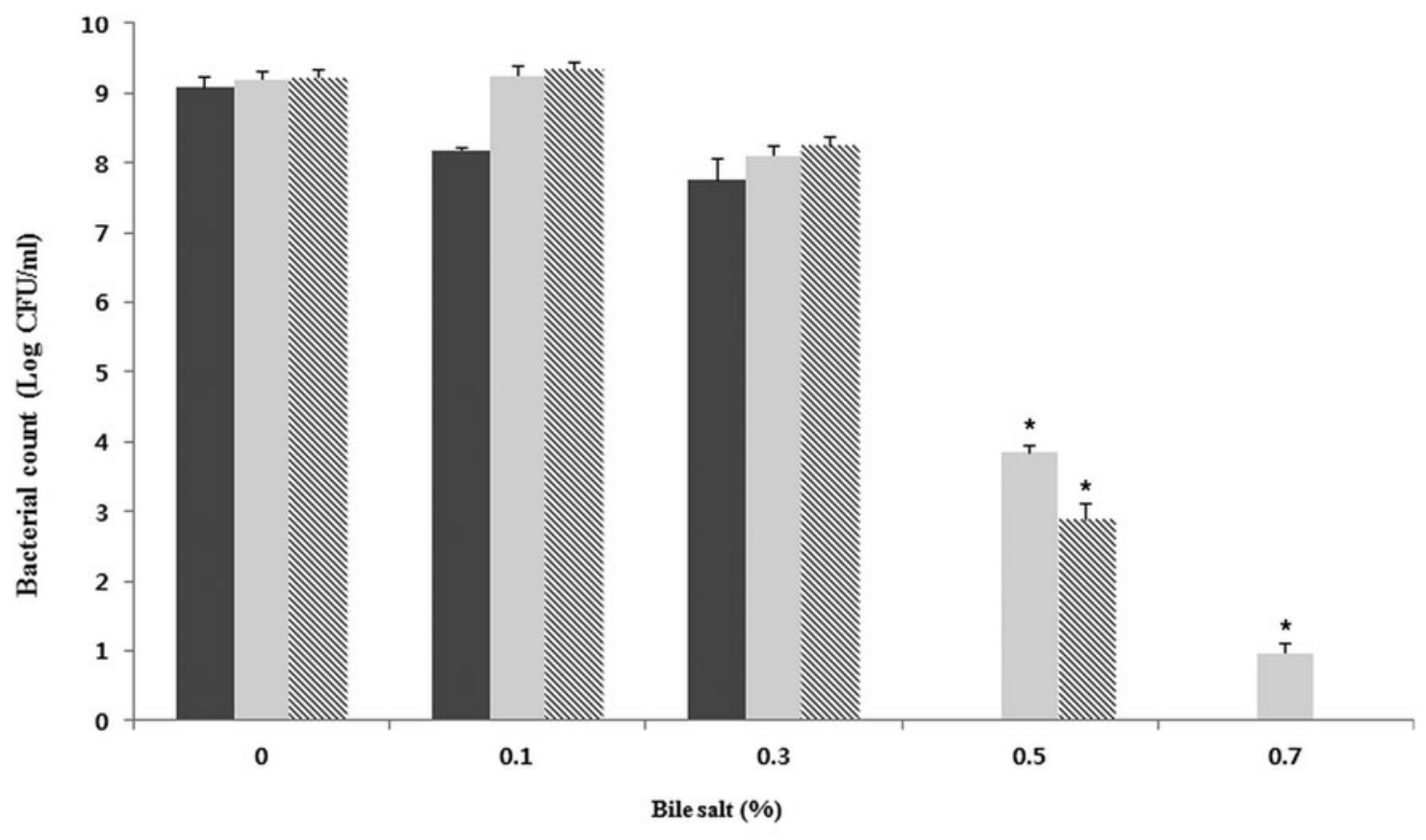

$\square$ L. Rhamnosus Mei

$\square$ L. Plantarum LB818

$\mathbb{Q}$ L. Johnsonii

FIG. 2. Effect of bile salts on Lactobacillus rhamnosus Mei, Lactobacillus plantarum LB818, Lactobacillus johnsonii, viability at different concentrations $(0 \%, 0.1 \%, 0.3 \%, 0.5 \%$, and $0.7 \%)$. Data are shown as a number of surviving bacteria at high concentration of bile salts. Data were represented as mean $\pm \mathrm{SD}(n=3)$. The value were compared using Student's $t$-test $(* P<.05)$. Error bars represent SD. SD, standard deviation.

HFD+LB818-treated animals (Fig. 4A). At the end of the 8th week, the body weight, liver, intestine, and total fat weight of HFD-fed mice were significantly higher $(P<.05)$ compared with those in the ND group, indicating successful HFD-induced obesity in the mice. However, treatment of HFD-fed animals with LB818 showed a significant $(P<.005)$ decrease in total body weight gain (Fig. 4A, B). In contrast, HFD-fed animals treated with a positive control group (HFD+SMV) caused a significant $(P<.005)$ decrease in total body weight gain as compared with the HFD-fed animals. The liver, intestinal fat, and total fat weight were also significantly $(P<.05)$ increased in HFD-fed mice compared with the ND control group. However, the LB818treated group had significantly $(P<.05)$ attenuated the liver weight and total fat weight, and intestinal fat weight (Fig. 4C-E), whereas HFD-fed animals in the positive control group (HFD+SMV) had a significant $(P<.005)$ decrease in the liver, intestinal fat, and total fat weight
A L. Rhamnosus Mei

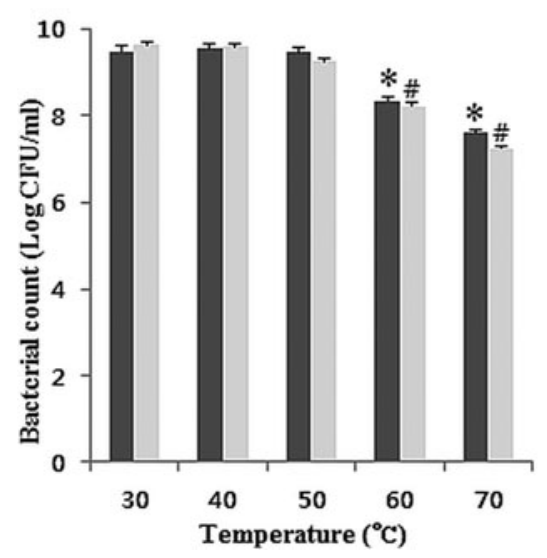

B L. Plantarum LB818

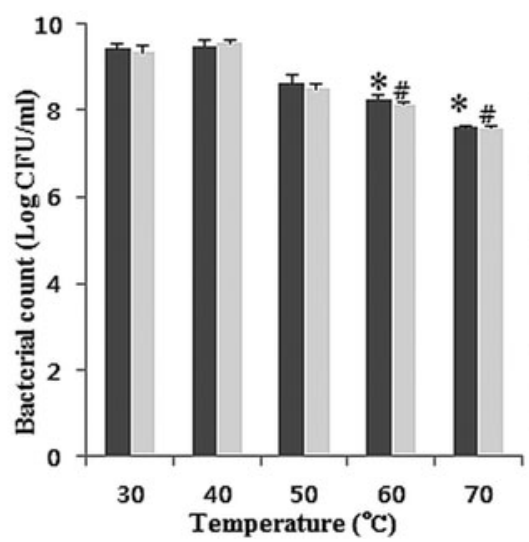

\section{L. Johnsonii}

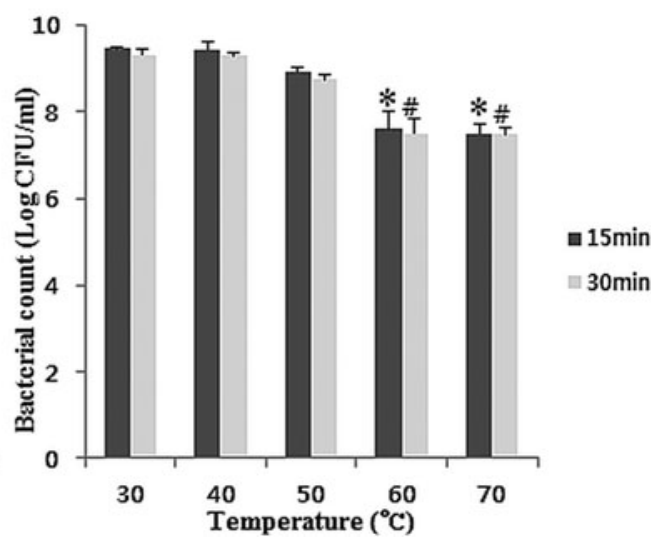

FIG. 3. The effect of high temperatures $\left(30^{\circ} \mathrm{C}, 40^{\circ} \mathrm{C}, 50^{\circ} \mathrm{C}, 60^{\circ} \mathrm{C}\right.$, and $\left.70^{\circ} \mathrm{C}\right)$ on the survival of LP LB818. (A) Lactobacillus rhamnosus Mei, (B) LP LB818, and (C) Lactobacillus johnsonii. Data are represented as mean \pm SD $(n=3)$. The values were compared using Student's $t$-test $\left({ }^{*} P<.05\right.$ and $\left.{ }^{\#} P<.05\right)$. The statistical significance compared with temperatures $30^{\circ} \mathrm{C}$. Error bars represent SD. 

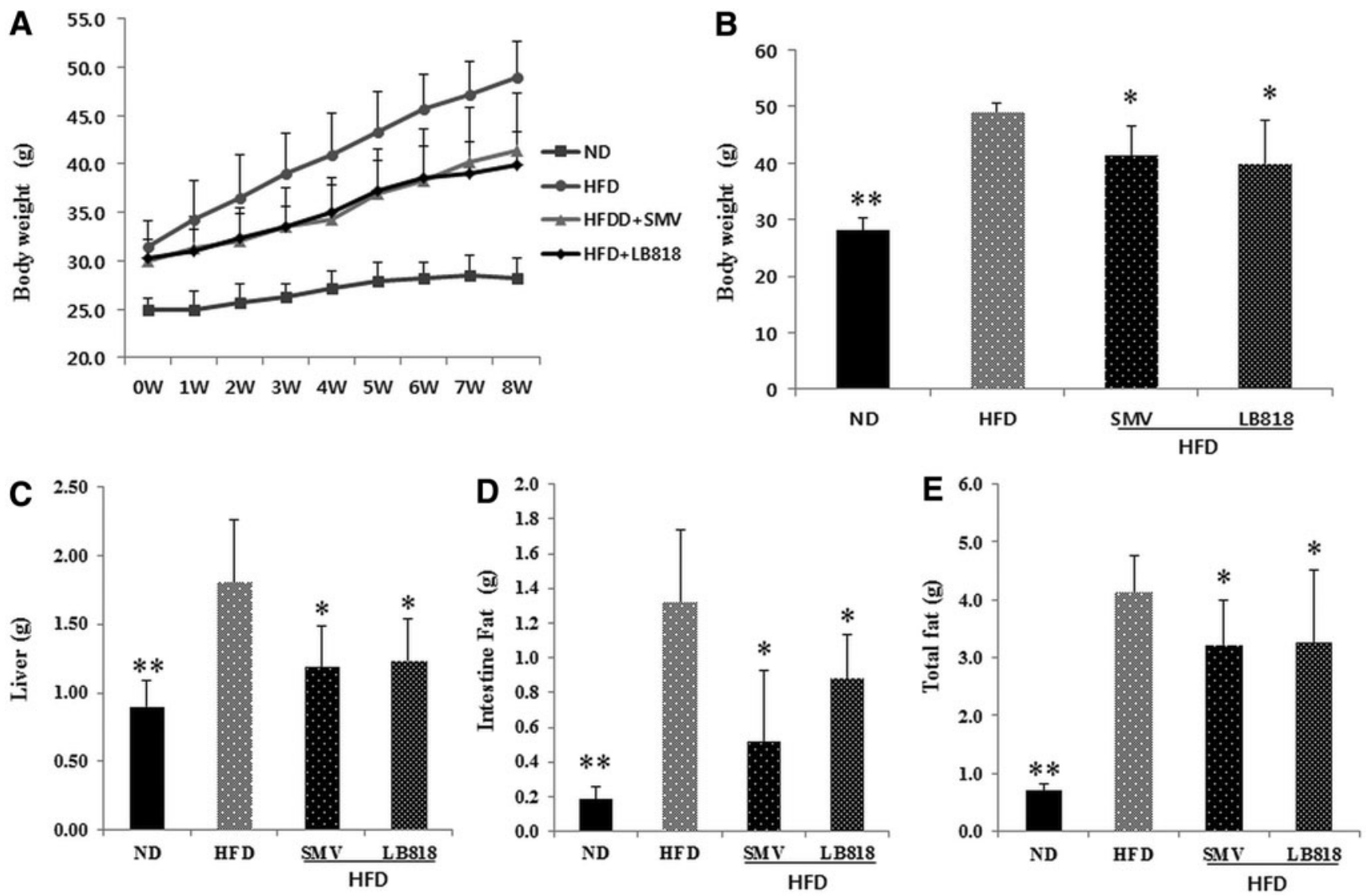

FIG. 4. Effect of LP LB818 on the body, liver, and fat weights in HFD-fed C57BL/6 mice: (A) the total body weight of ND, HFD, HFD+SMV, and Lactobacillus plantarum LB818 (HFD+LB818) mice during entire experimental period (0-8 weeks); (B) Body weight of animals at 8th week of treatments; (C) liver weight of ND, HFD, HFD+SMV and LB818; (D) intestinal fat of ND, HFD, HFD+SMV and LB818; (E) total fat of ND, HFD, HFD+SMV, and LB818. Data are represented as mean \pm SD $(n=7)$. The values were compared using Student's $t$-test $(* * P<.005, * P<.05)$. The statistical significance was compared with HFD control group. Error bars represent SD. HFD, high-fat diet; ND, normal diet; SMV, simvastatin.

compared with the HFD-fed mice. Accordingly, our results showed that LB818 can successfully prevent body weight gain and fat deposition in HFD-induced animals.

\section{Effects of LB818 treatment on biochemical parameters in HFD-induced obese mice}

After 8 weeks of treatment, in comparison with those fed ND, HFD-fed animals displayed significantly higher levels $(P<.05)$ of serum AST, ALT, TC, and LDL (Fig. 5A-C, E), but did not show a significant effect for TG concentrations (Fig. 5D) and significant $(P<.05)$ decreased level of serum HDL (Fig. 5F). However, treatment of HFD-fed mice with LB818 and SMV significantly decreased the levels of serum ALT, AST, TC, and LDL $(P<.05)$, but the serum TG level did not show a significant decrease in either LB818 or SMVtreated HFD-fed mice (Fig. 5D). In addition, the HDL levels were significantly $(P<.05)$ increased in HFD+LB818treated mice and HFD+SMV-treated mice (Fig. 5E). Fasting blood sugar levels were found to be significantly elevated $(P<.05)$ in the HFD-fed group compared with those in ND. However, the fasting blood sugar levels were significantly attenuated $(P<.05)$ in HFD-fed animals treated with LB818 and SMV-treated mice versus HFD-fed mice exhibited no significant difference in fasting blood sugar (Fig. 5G).

\section{LB818 improved HFD-induced changes and fat deposition in liver tissue of HFD-induced obese mice}

After 8 weeks of treatment, histopathological evaluation of the liver tissues and H\&E-stained tissue sections indicated normal lobular architecture, with the emergence of only a few small-sized fat droplets, in ND-fed animals. In contrast, large-sized fat droplets were noticed in the livers of HFD-fed animals that had a substantial deposition of fat, thereby indicating a state of fatty liver. However, liver tissues seemed to be normal, with few or even an absence of deposition of fat droplets in HFD-fed mice treated with LB818. Similar results were observed in animals treated with SMV (positive control) (Fig. 6A).

Furthermore, histological evaluation of frozen liver tissue sections of ND animals stained with Oil Red-O displayed normal lobular architecture of the liver tissue, with minor appearance of large fat droplets. In contrast, the livers of 

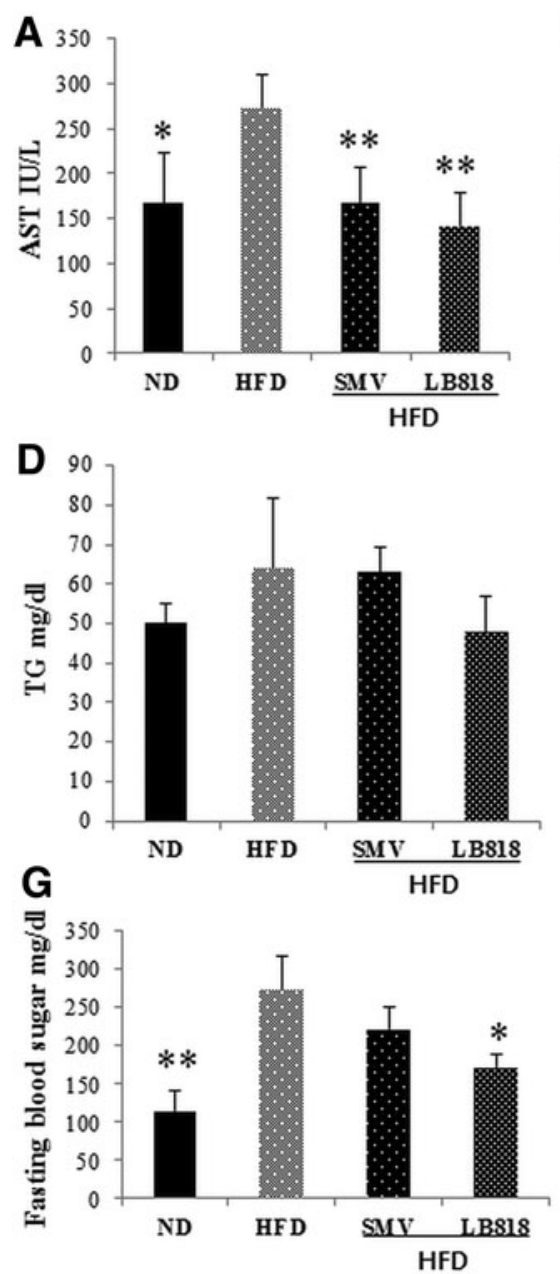
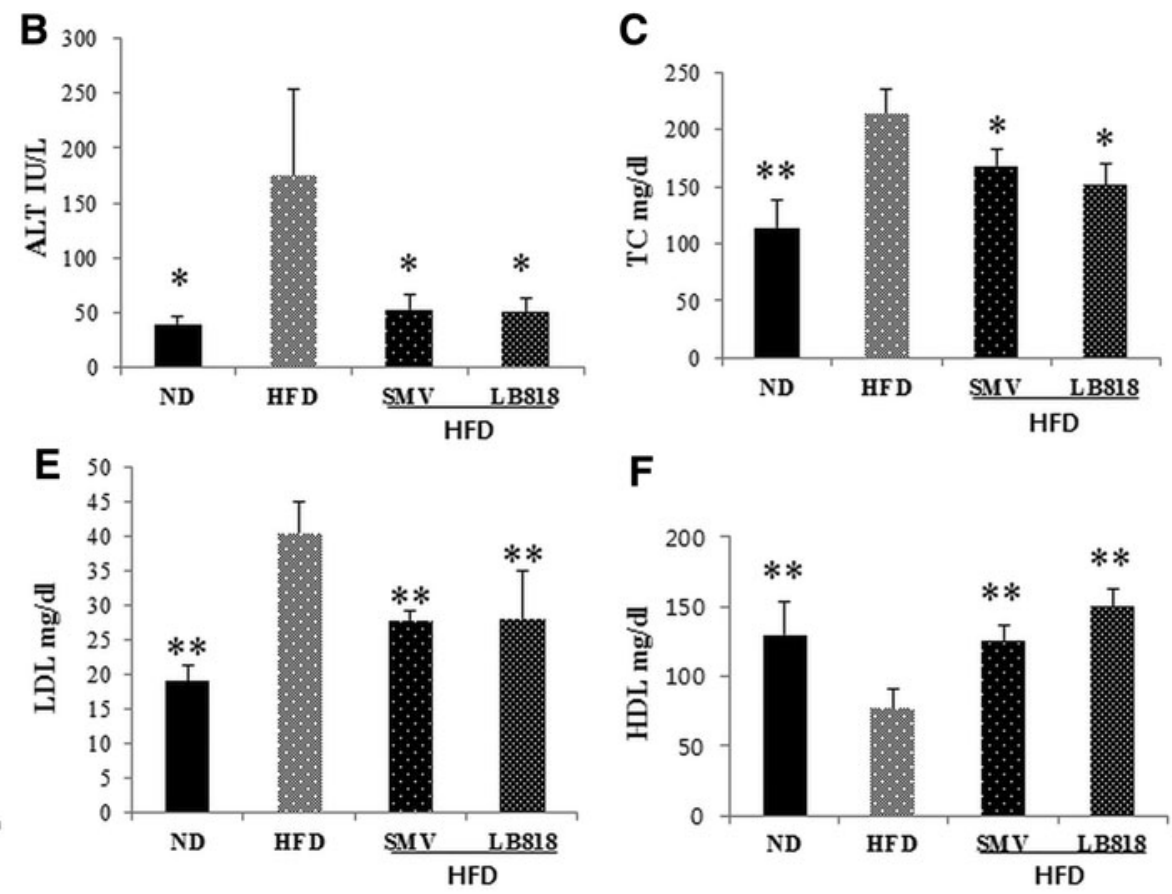

$\mathbf{F}$

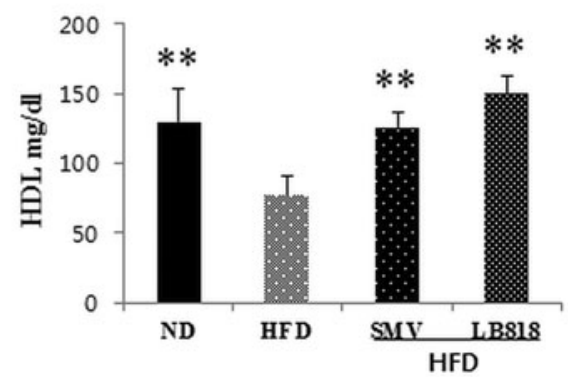

FIG. 5. Effect of Lactobacillus plantarum LB818 on and fasting blood sugar in HFD-fed C57BL/6 mice. The serum biochemical parameter of ND, HFD, HFD+SMV, and Lactobacillus plantarum LB818 (HFD+LB818)-treated animals after 8 weeks. (A) AST, (B) ALT, (C) TC, (D) TG, (E) LDL, (F) HDL, and (G) fasting blood sugar. Data are represented as mean \pm SD $(n=7)$. The values were compared by Student's $t$-test $(* * P<.005, * P<.05)$. The statistical significance compared with HFD group. Error bars represent SD. ALT, alanine transaminase; AST, aspartate transaminase; HDL, high-density lipoprotein; LDL, low-density lipoprotein; TC, total cholesterol; TG, triglyceride.

HFD-fed mice showed an aberrant histological structure dominated by abundant large fat droplets indicating the state of fatty liver. However, minimal numbers of small fat droplets were observed in HFD-fed mice when treated with LB818. Similar results were observed in animals treated with SMV (Fig. 6B).

\section{Effect of LB818 treatment on gut microbial population in HFD-induced obese mice}

The population of the intestinal microbial community was determined using quantitative real-time PCR (Fig. 7). The comparative abundance of bacteria of the phylum Bacteroidetes and genera Akkermansia, and Lactobacillus and Bifidobacteria was found to be decreased in HFD-fed animals compared with the ND group (Fig. 7A, D-F). In contrast, the load of phylum Firmicutes was significantly increased in the HFD-fed group compared with the ND group (Fig. 7B). However, the relative abundance of lactobacilli significantly $(P<.05)$ increased in the HFD-fed group treatment with HFD+LB818, whereas Akkermanasia and Bifidobacteria species in the HFD-fed group were insignificantly increased upon treatment with HFD+LB818. In addition, the relative abundances of Akkermanasia significantly increased $(P<.05)$, whereas Lactobacillus increased but not significantly in HFD-fed mice treated with HFD+SMV. However, the relative abundances of Bifidobacteria were found to decrease in HFD-fed mice treated with HFD+SMV. In contrast, the relative abundance of Bacteroidetes in the HFD-fed mice did not show a significant increase in response to the treatments with HFD+LB818, whereas the respective abundance of Bacteroidetes in HFD-fed group was significantly increased $(P<.05)$ in response to treatment with SMV. In contrast, the relative abundance of Firmicutes in HFD-fed animals was significantly $(P<.05)$ decreased when exposed to HFD+LB818 and insignificantly decreased 
A

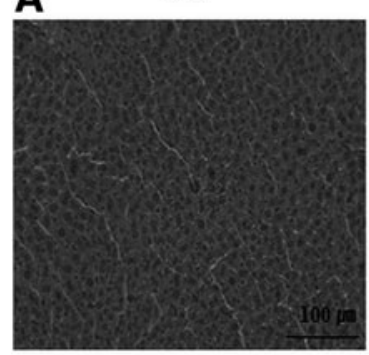

H\&E Staining

B

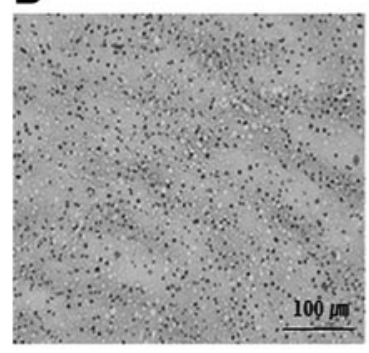

Oil Red O Staining
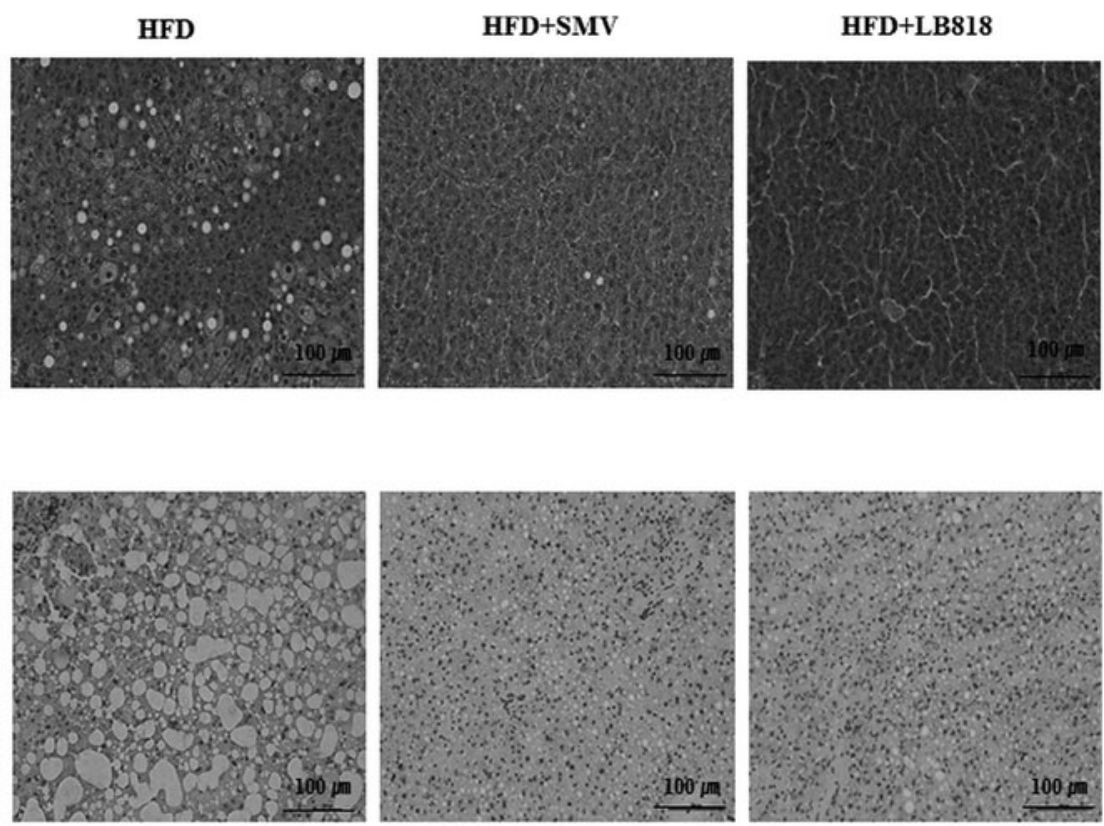

FIG. 6. Effect of Lactobacillus plantarum LB818 on HFD-induced histopathological changes of liver tissue observed with H\&E and Oil Red-O staining. (A) Representative image of liver tissue of ND, HFD, HFD+SMV, and Lactobacillus plantarum LB818 (HFD+LB818) stained with H\&E. (B) Representative image of liver tissue of ND, HFD, HFD+SMV, and Lactobacillus plantarum LB818 (HFD+LB818) stained with Oil red O. Pathophysiological examination of the liver tissue sections was performed under a light microscope with $200 \times$ magnification (scale bar $100 \mu \mathrm{m}$, arrows indicate lipid droplets). H\&E, hematoxylin and eosin.

when exposed to HFD+SMV. The Bacteroidetes:Firmicutes ratio was lower in the HFD-fed group compared with the ND group (Fig. 7C). However, the Bacteroidetes:Firmicutes ratio was higher in both the HFD+LB818- and HFD+SMVtreated groups.

\section{DISCUSSION}

Based on previous studies, ${ }^{22,23}$ we know that an acidic environment $(\mathrm{pH} 2.5-3.5)$ in the stomach and intestinal tract that contains $0.3 \%(\mathrm{w} / \mathrm{v})$ bile salts helps prevent pathogenic infections. Therefore, probiotic strains should be acidic and bile salt tolerant to survive in the stomach. Artificial gastric juice, bile acid salts, and high-temperature environments are all easily tolerated by LB818, as shown in Figures 1-3. According to a previous study, the survival rate of Lactobacillus plantarum KLDS1.0391 was $92.6 \%$ in the presence of pepsin $(0.3 \%)$, whereas $97.62 \%$ in the presence of bile salts $(0.3 \%) .{ }^{22}$ In our study, LB818 survived in both acidic and alkaline conditions, and therefore it could be expected that LB818 may survive in the human gastrointestinal tract environment. The LB818 strain displayed a strong tolerance to acidic conditions, and high survival rate in bile salt and high temperature conditions. Thus, these results signify the tolerance of LB818 strain to survive in the acidic environment of the stomach. ${ }^{24}$

Recently, the use of probiotics has emerged as an important platform for therapeutic and preventive effects on the development of metabolic disorders like obesity and diabe- tes. Several studies have demonstrated the role of probiotics against obesity and diabetes. ${ }^{25,26}$ Herein, we explored the anti-obesity therapeutic effect of LB818 using an HFDinduced obesity mice model.

The HFD-induced C57BL/6 mouse is a universal obesity animal model for studying the interplay mechanism of obesity. ${ }^{27}$ In this study, we administrated oral supplements of LB818 at $10^{9}$ CFU once a day, similar to previously reported studies. ${ }^{28,29}$ The HFD-fed mice demonstrated significantly higher weight gain, compared with the ND-fed mice, which revealed that diet-induced obesity was successfully achieved, whereas HFD fed along with oral administration of LB818 did not exhibit any weight gain change, which revealed that LB181 possesses anti-obesity efficacy. A similar anti-obesity effect was also shown by SMV on HFD-fed mice.

Increased adipose tissue is the first characteristic of obesity, which indicates an increased number and size of adipocytes. According to the metabolic requirements of an organism, adipose tissue plays an important role in energy and appetite regulation. ${ }^{30,31}$ Furthermore, in this study, there was a significant decrease in liver weight, intestinal fat weight, and total fat in HFD+LB818-treated mice compared with that in HFD-fed mice, suggesting that LB818 effectively reduced proliferation of adipose tissues. Increased fat weights positively correlated with increased body weights. In the HFD-fed group, HFD+SMV-treated mice also showed significantly decreased liver weight, intestinal fat (visceral fat) weight, and total fat compared with the 

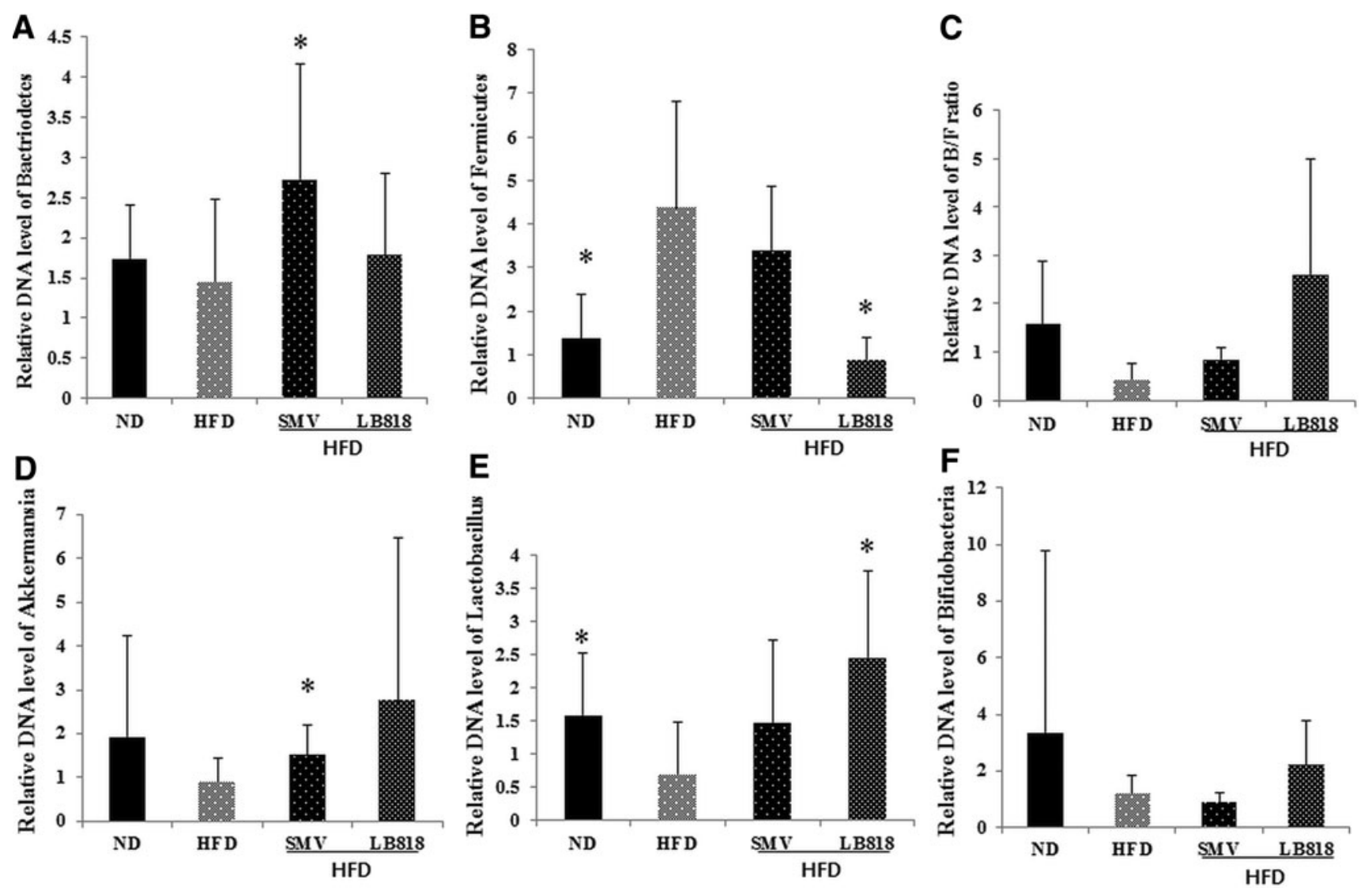

FIG. 7. The effect of Lactobacillus plantarum LB818 on relative abundance of intestinal microbes in stools of HFD-fed mice. The relative abundance of different gut flora was quantified by quantitative reverse transcription-polymerase chain reaction in animals fed ND, HFD, HFD+SMV, and HFD+LB818. Relative abundance of phyla (A) Bacteroidetes, (B) Firmicutes; (C) Bacteroidetes/Firmicutes ratio. Relative abundance of genera (D) Akkermansia, (E) Lactobacillus, and (F) Bifidobacteria. The results are expressed as normalized fold values relative to the normal group. Data are represented as mean $\pm \operatorname{SD}(n=7)$. The results were compared by Student's $t$-test $(* P<.05)$. The statistical significance compared with HFD group. Error bars represent SD.

HFD-fed mice. It can be concluded that the LB818 effect repressed body weight gain by suppressing the growth of adipose tissue mass. ${ }^{9}$

In the serum lipid profile of this study, HFD-fed mice showed elevated serum TC, TG, and AST/ALT levels, and a pronounced decrease in serum HDL level was observed in the HFD group compared with the ND group. However, the serum glucose level was significantly reduced with the treatment of LB818 in HFD-fed mice, producing a hypoglycemic effect. These results suggest a beneficial effect of LB818 on glucose homeostasis and lipid metabolism in HFD-induced obesity. Moreover, after treatment with either LB818 or SMV, the levels of serum biochemical profile, including TC, TG, HDL, ALT, and AST in HFD-fed mice were restored. Based on these results, it is conceivable that LB818 and SMV improve the serum biochemical parameters in HFD-induced obesity. Recent studies revealed that SMV treatment results in a marked improvement in serum and hepatic lipids and enzymes profiles, including TG, TC, LDL, and HDL in HFD-induced obese rats or mice. ${ }^{32-34}$ Collectively, these results indicate that LB818 exerts its anti-diabetic effect by combating body weight gain, reduces fat accumulation, by glucose homeostasis. Moreover,
LB818 treatment showed marked improvement in hepatocyte morphology and less accumulation of lipid in HFD-fed mice; similar effects with treatment by SMV further support the anti-obesity efficacy of LB818.

Previous findings revealed that an alteration in the gut bacterial population may be linked to obesity. ${ }^{35-42}$ Growing evidence of clinical and animal studies has shown that obese subjects with insulin resistance are characterized by modified gut microbiota composition, particularly an increase in Firmicutes:Bacteroidetes ratio compared with that of healthy subjects. ${ }^{39,40}$

To further investigate the alteration in the relative abundance of gut microbiota in HFD-fed mice by treatment with LB818, mouse stool samples were used to quantify intestinal flora using qRT-PCR. Our results indicated that feeding HFD to mice altered the relative abundance of gut microbial populations. Alterations in the relative abundance of gut microbiota populations at the levels of phylum or genus in obesity have been previously investigated. ${ }^{41,42}$ Our study shows a similar pattern to that observed in previous studies, in which the number of Firmicutes is increased and the number of Bacteroidetes is decreased, and an imbalance in the Bacteroidetes:Firmicutes ratio and the stability of the Akkermansia 
population is shown to be linked to obesity. ${ }^{43-46} \mathrm{~A}$ decrease in the population of Bifidobacterium and Lactobacillus is also associated with obesity. ${ }^{47,48}$ In agreement with these studies, we found that the anti-obesity effects of both LB818 and SMV on HFD-fed mice were associated with a restoration of essential gut microbes.

\section{CONCLUSION}

This study demonstrates that LB818 suppressed body weight gain; reduced fat deposition; reduced the levels of TC, TG, AST, ALT, LDL, and fasting glucose; and elevated HDL levels. These data indicate that LB818 attenuates obesity in mice through the modulation of intestinal microbiota. Furthermore, LB818 alters the spreading of intestinal flora and restores the respective abundances of essential bacteria, including Akkermansia, Lactobacillus, and Bifidobacterium, and maintains a balance in the Bacteroidetes:Firmicutes ratio, thereby preventing an imbalance of the gut microbiota in obese mice.

\section{AUTHOR DISCLOSURE STATEMENT}

No competing financial interests exist.

\section{FUNDING INFORMATION}

This work was financially supported by Small and Medium Business Administration and Lee's Biotech. Co., Ltd. Company, Republic of Korea.

\section{REFERENCES}

1. Power ML, Schulkin J: Sex differences in fat storage, fat metabolism, and the health risks from obesity: Possible evolutionary origins. Br J Nutr 2008;99:931-940.

2. Guh D, Zhang W, Bansback $\mathrm{N}$, et al:: The incidence of comorbidities related to obesity and overweight: A systematic review and meta-analysis. BMC Public Health 2009;25:9:88.

3. Caricilli AM, Saad MJ: Gut microbiota composition and its effects on obesity and insulin resistance. Curr Opin Clin Nutr Metab Care 2014;17:312-318.

4. Tremaroli V, Bäckhed FV: Functional interactions between the gut microbiota and host metabolism. Nature 2012;489:242-249.

5. Ley RE: Obesity and the human microbiome. Curr Opin Gastroenterol 2010;26:5-11.

6. Cani PD, Delzenne NM: Gut microflora as a target for energy and metabolic homeostasis. Curr Opin Clin Nutr Metab Care 2007; 10:729-734.

7. Kaoutari AE, Armougom F, Gordon JI, et al.: The abundance and variety of carbohydrate active enzymes in the human gut microbiota. Nat Rev Microbio 2013;11:497-504.

8. Aguirre M, Venema K: The art of targeting gut microbiota for tackling human obesity. Genes Nutr 2015;10:472.

9. Patterson E, Ryan PM, Cryan JF, et al.: Gut microbiota, obesity and diabetes. Postgrad Med J 2016;92:286-300.

10. Boulangé CL, Neves AL, Chilloux J, et al.: Impact of the gut microbiota on inflammation, obesity, and metabolic disease. Genome Med 20168:42.
11. Gill H, Prasad J: Probiotics, immunomodulation, and health benefits. Adv Exp Med Biol 2008;606:423-454.

12. Kumar M, Kumar A, Naqpal R, et al.: Cancer preventing attributes of probiotics: An update. Int J Food Sci Nutr 2010;61:473496.

13. Menniqen R, Bruewer M: Effect of probiotics on intestinal barrier function. Ann N Y Acad Sci 2009;1165:183-189.

14. Shin HS, Park SY, Lee DK, et al.: Hypocholesterolemic effect of sonication-killed Bifidobacterium longum isolated from healthy adult Koreans in high cholesterol fed rats. Arch Pharm Res 2010; 33:1425-1431.

15. Behnsen J, Deriu E, Sassone-Corsi M, et al.: Probiotics: Properties, examples, and specific applications. Cold Spring Harb Perspect Med 2013;3:a010074.

16. Park DY, Ahn YT, Huh CS, et al.: Dual probiotic strains suppress high $\mathrm{f}$ ructose-induced metabolic syndrome. World $J$ Gastroenterol 2013;19:274-283.

17. Bhathena J, Martoni C, Kulamarva A, et al.: Orally delivered microencapsulated live probiotic formulation lowers serum lipids in hypercholesterolemic hamsters. J Med Food 2009;12:310 319.

18. Lee K, Paek K, Lee HY, et al.: Antiobesity effect of trans-10, cis12-conjugated linoleic acid-producing Lactobacillus plantarum PL62 on diet induced obese mice. J Appl Microbiol 2007;103: 1140-1146.

19. Lye HS, Kuan CY, Ewe JA, et al.: The improvement of hypertension by probiotics: Effects on cholesterol, diabetes, renin, and phytoestrogens. Int J Mol Sci 2009;10:3755-3775.

20. Park JE, Oh SH, Cha YS: Lactobacillus plantarum LG42 isolated from gajami sik-hae decreases body and fat pad weights in dietinduced obese mice. J Appl Microbiol 2014;116:145-156.

21. Wang JH, Bose S, Kim HG, et al:: Fermented Rhizoma Atractylodis Macrocephalae alleviates high fat diet-induced obesity in association with regulation of intestinal permeability and microbiota in rats. Sci Rep 2015;5:8391.

22. Han Q, Kong B, Chen Q, et al.: In vitro comparison of probiotic properties of lactic acid bacteria isolated from Harbin dry sausages and selected probiotics. J Funct Food 2017;32:391-400.

23. Jeon EB, Son SH, Jeewanthi RKC, et al.: Characterization of Lactobacillus plantarum Lb41, an isolate from kimchi and its application as a probiotic in cottage cheese. Food Sci Biotechnol 2016;25:1129-1133

24. Maragkoudakis PA, Zoumpopoulou G, Miaris C, et al.: Probiotic potential of Lactobacillus strains isolated from dairy products. Int Dairy J 2006;16:189-199.

25. Kadooka Y, Sato M, Imaizumi K, et al.: Regulation of abdominal adiposity by probiotics (Lactobacillus gasseri SBT2055) in adults with obese tendencies in a randomized controlled trial. Eur $J$ Clin Nutr 2010;64:636-643.

26. Bordalo Tonucci L, Dos Santos KM, De Luces Fortes Ferreira CL, et al.: Gut microbiota and probiotics: Focus on diabetes mellitus. Crit Rev Food Sci Nutr 2017;57:2296-2309.

27. Wang CY, Liao JK: A mouse model of diet-induced obesity and insulin resistance. Methods Mol Biol 2012;821:421-433.

28. Sakai T, Taki T, Nakamoto A, et al.: Lactobacillus plantarum OLL2712 regulates glucose metabolism in C57BL/6 mice fed a high-fat diet. J Nutr Sci Vitaminol (Tokyo) 2013;59:144-147.

29. Park JE, Oh SH, Cha YS: Lactobacillus plantarum LG42 isolated from gajami sik-hae decreases body and fat pad weights in dietinduced obese mice. J Appl Microbiol 2014;116:145-156. 
30. Pichiah PBT, Moon HJ, Park JE, et al.: Ethanolic extract of sea buckthorn (Hippophae rhamnoides L) prevents high-fat diet induced obesity in mice through down-regulation of adipogenic and lipogenic gene expression. Nutr Res 2012;32:856-864.

31. Choe SS, Huh JY, Hwang, IJ, et al.: Adipose tissue remodeling: It's role in energy metabolism and metabolic disorders. Front Endocrinol (Lausanne) 2016;7:30.

32. Hoffman KB, Kraus C, Dimbil M, et al.: A survey of the FDA's AERS database regarding muscle and tendon adverse events linked to the statin drug class. PLoS One 2012;7:e42866.

33. Qian W, Cai X, Zhang X, et al.: Effect of Daisaikoto on expressions of SIRT1 and NF-kappaB of diabetic fatty liver rats induced by high-fat diet and streptozotocin. Yonago Acta Med 2016;59:149-158.

34. Cheon SY, Chung KS, Lee KJ, et al.: An HJ. HVC1 ameliorates hyperlipidemia and inflammation in $\mathrm{LDLR}^{-1-}$ mice. BMC Complement Altern Med 2017;17:222.

35. Wang JH, Bose S, Kim GC, et al.: Flos Lonicera ameliorates obesity and associated endotoxemia in rats through modulation of gut permeability and intestinal microbiota. PLoS One 2014;9:e86117.

36. Han K, Bose S, Kim YM, et al.: Rehmannia glutinosa reduced waist circumferences of Korean obese women possibly through modulation of gut microbiota. Food Funct 2015;6:2684-2692.

37. Lee SJ, Bose S, Seo JG, et al:: The effects of co-administration of probiotics with herbal medicine on obesity, metabolic endotoxemia and dysbiosis: A randomized double-blind controlled clinical trial. Clin Nut 2014;33:973-981.

38. Hussain A, Yadav MK, Bose S, et al.: Daesiho-tang is an effective herbal formulation in attenuation of obesity in mice through alteration of gene expression and modulation of intestinal microbiota. PLoS One 2016;11:e0165483.

39. Tilg H, Kaser A: Gut microbiome, obesity, and metabolic dysfunction. J Clin Invest 2011;121:2126-2132.

40. Tremaroli V, Bäckhed F: Functional interactions between the gut microbiota and host metabolism. Nature 2012;489:242-249,

41. Turnbaugh PJ, Hamady M, Yatsunenko T, et al:: A core gut microbiome in obese and lean twins. Nature 2009;457:480-484.

42. Turnbaugh PJ, Ley RE, Mahowald MA, et al.: An obesityassociated gut microbiome with increased capacity for energy harvest. Nature 2006;444:1027-1023.

43. Everard A, Cani PD: Diabetes, obesity and gut microbiota. Best Prac Res Clin Gastroenterol 2013;27:73-83.

44. Ley RE, Bäckhed F, Turnbaugh $\mathrm{P}$, et al: Obesity alters gut microbial ecology. Proc Natl Acad Sci U S A 2005;102:1107011075.

45. Ganesh BP, Klopfleisch R, Loh G, et al:: Commensal Akkermansia muciniphila exacerbates gut inflammation in salmonella typhimurium-infected gnotobiotic mice. PLoS One 2013;8: e74963.

46. Ley RE, Turnbaugh PJ, Klein S, et al.: Microbial ecology: Human gut microbes associated with obesity. Nature 2006;444: 1022-1023.

47. Lecomte V, Kaakoush NO, Maloney CA, et al:: Changes in gut microbiota in rats fed a high fat diet correlate with obesityassociated metabolic parameters. PLoS One 2015;10:e0126931.

48. Sanz Y, Rastmanesh R, Agostonic C: Understanding the role of gut microbes and probiotics in obesity: How far are we? Pharmacol Res 2013;69:144-155. 